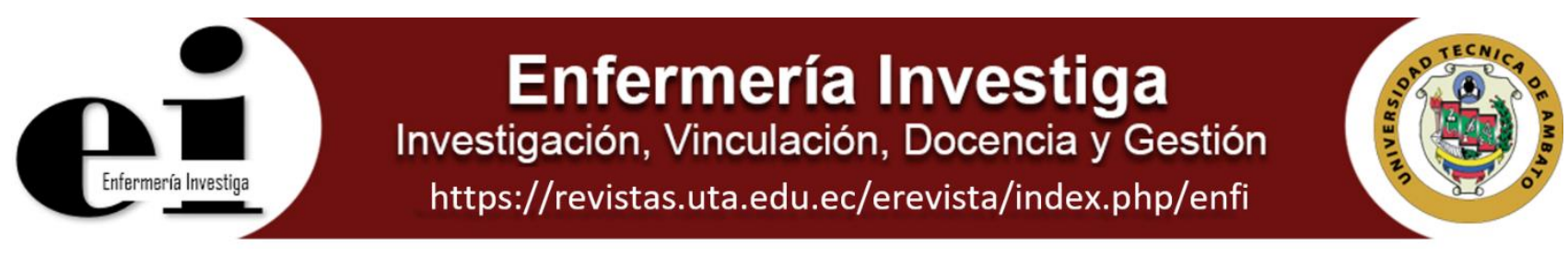

\title{
CALIDAD DE VIDA DE LOS CUIDADORES DE PERSONAS CON ENFERMEDAD DE ALZHEIMER: REVISIÓN INTEGRATIVA.
}

\section{QUALITY OF LIFE OF CAREGIVERS OF PEOPLE WITH ALZHEIMER'S DISEASE: A COMPREHENSIVE REVIEW}

\author{
Rosa Herminia Pastuña Doicela https://ortid.orq/0000-0003-2008-7855
}

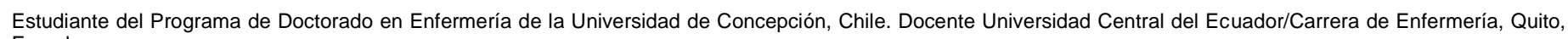
Ecuador.

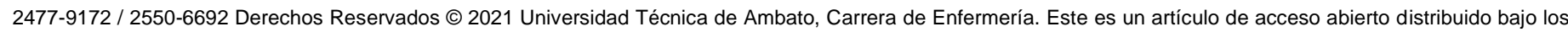

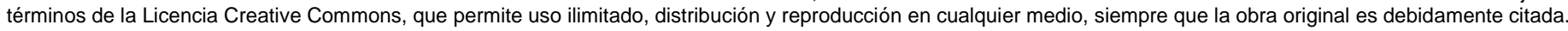

Recibido: 14 de septiembre 2021

Aceptado: 12 de octubre 2021

\section{RESUMEN}

Introducción: En la actualidad un gran número de adultos mayores es vulnerable a enfermedades crónicas y otras afecciones como la enfermedad de Alzheimer, por lo que el cuidado de estas personas representa un desafío. Objetivo: Analizar la calidad de vida de los cuidadores de personas con la enfermedad de Alzheimer. Métodos: Se realizó una revisión integrativa de la literatura científica, con una pregunta de investigación con el PICO: ¿Cuál es la Calidad de Vida de los cuidadores de personas con enfermedad de Alzheimer?, posteriormente se inició la búsqueda de artículos científicos en las siguientes bases de datos: Pub Med; Wef of Science (WOS); Bvs; SciELO y Scopus. Utilizando los siguientes DeCS: "Quality of life", "Caregivers" y "Alzheimer Disease", identificados inicialmente 343 artículos, de los cuales 15 fueron elegibles y 7 fueron seleccionados porque cumplieron con los criterios de inclusión y exclusión. Resultados: El cuidado de la persona con enfermedad de Alzheimer recae mayormente en las familias. La mayor parte de cuidadoras/es son mujeres, el cuidado es integral, continuo, con desgaste físico y psicológico, estos factores afectan negativamente en su calidad de vida $y$ compromete su salud. Conclusiones: La calidad de vida del cuidador de personas con enfermedad de Alzheimer se ve comprometida, debido al aparecimiento de afecciones físicas, psicológicas, sociales y económicas, derivadas por el desgaste y la sobrecarga.

Palabras clave: calidad de vida; cuidadores; enfermedad de Alzheimer; Atención de Enfermería

\section{ABSTRACT}

Introduction: The new demographic transition because of the phenomenon of globalization has generated improvements in health care. Despite this, today a large number of older adults are vulnerable to chronic diseases and other conditions such as Alzheimer's disease, so caring for these people represents a challenge for their caregivers. Objective: Analyze the quality of life of caregivers of people with Alzheimer's disease. Methods: An integrative review of the scientific literature was carried out, for the formulation of the research question, the PICO format was used: What is the Quality of Life of caregivers of people with Alzheimer's disease? Subsequently, the search for scientific articles was started in the following databases: Pub Med; Wef of Science (WOS); Bvs; SciELO and Scopus. Using the following DeCS: "Quality of life", "Caregivers" and "Alzheimer Disease", associated with the Boolean operator "AND". 343 articles were initially identified, of which 15 were eligible and 7 were selected because they met the inclusion and exclusion criteria. Results: The care of the person with Alzheimer's disease falls mostly in families. The majority of caregivers are women, the care is integral, continuous, with physical and psychological wear, and these factors negatively affect their quality of life and compromise their health. Conclusions: The quality of life of the caregiver of people with Alzheimer's disease is compromised, due to the appearance of physical, psychological, social and economic conditions, derived from wear and tear and overload. Therefore, making the needs and problems derived from their visible role can contribute significantly to the development of comprehensive care interventions in this vulnerable population

Keywords: quality of life; caregivers; Alzheimer disease; Nursing care 


\section{INTRODUCCIÓN}

La nueva transición demográfica como resultado del fenómeno de la globalización ha generado mejoras en la atención de la salud, principalmente por el desarrollo tecnológico el cual ha contribuido de manera significativa en el tratamiento, cuidados y rehabilitación de enfermedades $(1,2)$, este importante avance ha permitido un crecimiento exponencial de personas mayores a escala mundial (3), alcanzando hoy una esperanza de vida promedio de 80,7 años para las mujeres y de 74,9 años para los hombres $(4,5)$.

En la actualidad un gran número de adultos mayores gozan de una vida activa y saludable, pero al mismo tiempo un grupo importante es vulnerable a enfermedades crónicas y otras afecciones, una población envejecida es más susceptible a enfermedades crónicas no transmisibles (6), donde se incluyen las demencias, a pesar del progreso científico la mayor parte de demencias no son curables (7).

La Enfermedad de Alzheimer (EA) es el tipo de demencia más frecuente, se presenta en un 60 a $80 \%(1,8)$, en los últimos años se ha incrementado su incidencia de manera rápida en todo el mundo8, lo que constituye un problema de salud pública y social a escala global (9). Actualmente existen 47 millones de personas viviendo con este padecimiento en el mundo (7).

Como enfermedad neurodegenerativa produce un deterioro en la autonomía, por la pérdida gradual e irreversible de la capacidad cognitiva y funcional9,10, lo cual imposibilita el desenvolvimiento normal del individuo, que repercute de manera directa e importante en la familia, por el alto nivel de discapacidad y dependencia, que demanda mayor supervisión y cuidados continuos que se tornan cada vez más complejos a medida que evoluciona la enfermedad (11).

La EA es devastadora no sólo para la persona enferma sino también para sus cuidadores y toda la estructura familiar en general $(9,11)$, la familia constituye el pilar fundamental para el acompañamiento, apoyo y cuidado del enfermo, cerca del $94 \%$ de los cuidadores corresponde a un familiar directo, de estos la mayor parte del cuidado recae en un solo miembro familiar, de los cuales el $90 \%$ son mujeres cuidadoras (12).

Cada familia percibe la enfermedad de manera particular y experimenta modificaciones constantes en su organización, funcionamiento, redistribución de roles familiares y con un elevado nivel de estrés a medida que avanza la enfermedad, todo esto puede ocasionar alteraciones psicológicas, estrés emocional crónico, desgaste físico, descuido personal y aislamiento social, lo cual conlleva un incremento en la necesidad de asistencia sanitaria y pérdida gradual de la calidad de vida de los cuidadores $(12,13)$.

Según la OMS la calidad de vida se define como "la percepción del individuo de su posición en la vida, en el contexto de la cultura y sistemas de valores en los cuales vive y en relación a sus objetivos, expectativas, estándares y preocupaciones" (13). Este concepto tiene un interés creciente, especialmente en enfermedades crónicas como la enfermedad de Alzheimer, cuando se carece de una cura para la enfermedad y desencadena en los cuidadores un sufrimiento silencioso en desmedro de su bienestar (14).

La preocupación por la salud y calidad de vida del cuidador debe abordarse de manera prioritaria por su gran relevancia, debido a que el cuidado de la persona enferma genera sobrecarga, estrés, largas horas de atención y cuidado físico exigente15, lo cual desencadena alteraciones nocivas en su bienestar que están asociadas al cuidado como: depresión, ansiedad, mayor uso de medicamentos psicotrópicos, disfunción inmunológica, todos estos factores impactan de manera negativa en la calidad de vida de los cuidadores (16), por lo expuesto anteriormente el objetivo de la presente revisión es analizar la calidad de vida de los cuidadores de personas con la enfermedad de Alzheimer.

\section{MÉTODOS}

El proceso metodológico de este estudio se basó en una revisión integrativa de la literatura científica, lo cual está recomendado actualmente por la Práctica de Enfermería Basada en Evidencias, que cuenta con un método de trabajo establecido en cinco fases contiguas. En la primera fase se formula la pregunta, interrogante o fenómeno de estudio que se desea resolver. En la segunda fase se de buscar la mejor evidencia para responder a la interrogante de investigación. La tercera fase corresponde a la evaluación crítica de la evidencia científica encontrada. La fase cuatro corresponde a integrar la evidencia y la quinta fase se refiere a la evaluación de los resultados (17).

Para la formulación de la pregunta de investigación se utilizó el formato PICO, incluyendo tres de sus componentes (PICO): ¿Cuál es la Calidad de Vida de los cuidadores de personas con enfermedad de Alzheimer?

Se realizó una revisión de la literatura científica en las principales bases de datos. Las bases de datos consultadas fueron: Pub Med, Wef of Science, BVS, SciELO y Scopus; para las cuales se utilizaron los siguientes descriptores: Quality of life; Caregivers; Alzheimer Disease; Nursing Care, en español, inglés y portugués, conjugados con el operador booleano AND, por las características de la investigación.

Para la búsqueda de la literatura científica se consideraron los siguientes criterios de inclusión y exclusión:

Criterios de inclusión: Estudios primarios; de texto completo; en idioma inglés, español y portugués; con un tiempo de 5 años: 2014 -2018; y que sean cuidadores de personas con enfermedad de Alzheimer.

Criterios de exclusión: Investigaciones secundarias como metaanálisis y revisiones sistemáticas, estudios realizados por otros profesionales que no pertenezcan a la disciplina de enfermería y artículos cuyos textos completos no estén disponibles en la web.

Los resultados de la búsqueda de literatura científica arrojaron un total de 343 artículos. Después de la evaluación inicial se excluyeron 328 artículos, ya que no guardaban relación con el tema de revisión, no estaban disponibles a texto completo, no eran estudios primarios, estaban repetidos y eran de otras disciplinas. 15 estudios cumplieron con los criterios para la revisión de texto completo. Se realizó la lectura crítica para evaluar la validez, fiabilidad, relevancia y pertinencia de los artículos seleccionados. De los cuales 8 fueron eliminados posteriormente por no contemplar en su estudio la calidad de vida de los cuidadores de personas con enfermedad de Alzheimer. Finalmente, se incluyó un total de 7 documentos que reunieron los criterios establecidos para esta revisión.

La revisión cumplió con los principios éticos, respetando los derechos de autor, realizando las citaciones específicas y dando crédito a los autores revisados. 
Figura $N^{\circ} 1$. Diagrama que ilustra las diferentes fases de búsqueda y selección de estudios.

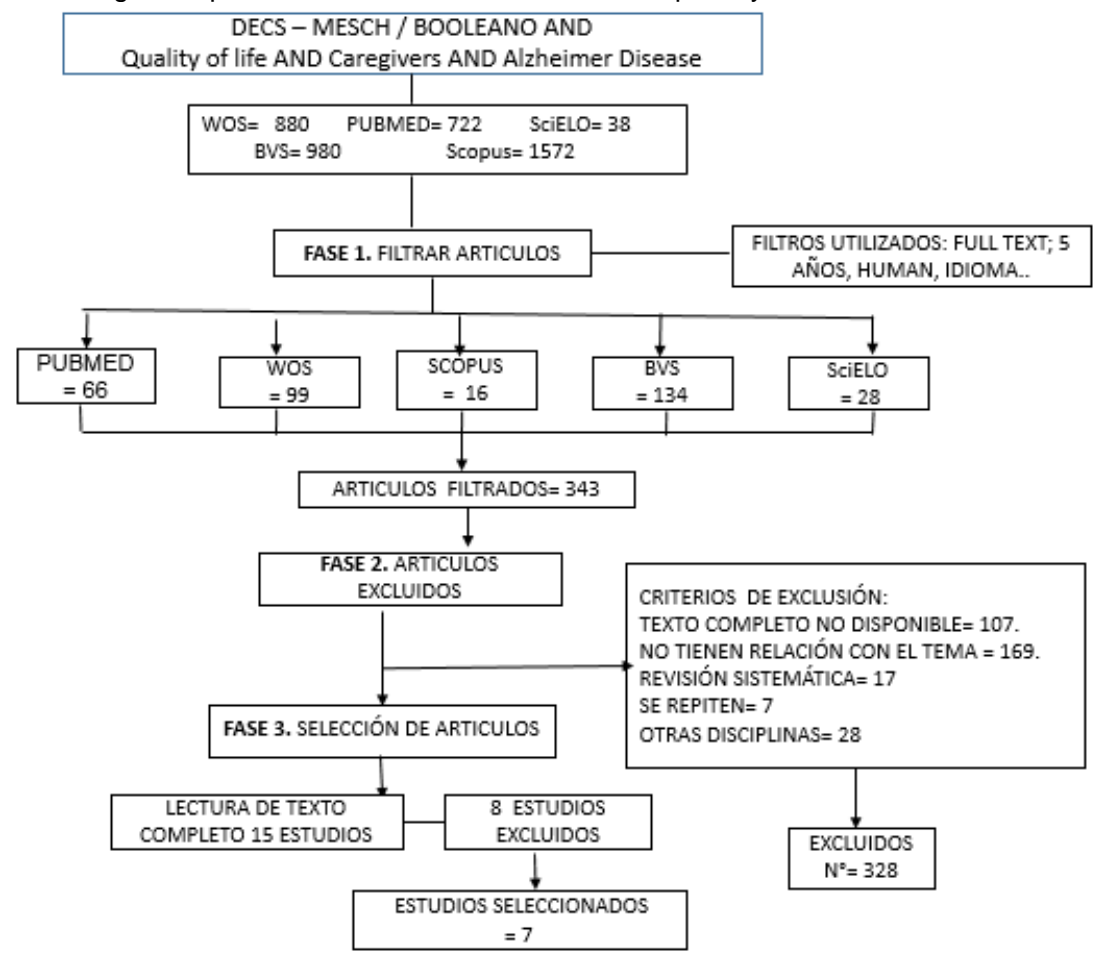

ELABORADO POR: LA AUTORA

\section{RESULTADOS.}

Con una línea base de 7 estudios que se relacionan con la temática, se procedió a sintetizarlos y analizarlos. Los artículos que compusieron el corpus de este estudio, se presentan en la Tabla №1.

Tabla $N^{\circ}$ 1. Artículos científicos seleccionados para la revisión.

\begin{tabular}{|c|c|c|c|c|}
\hline Título & $\begin{array}{l}\text { Autores } \\
\text { País/Año }\end{array}$ & $\begin{array}{ll}\text { Diseño } & \text { de } \\
\text { investigación } & \end{array}$ & $\begin{array}{ll}\text { Instrumentos } \\
\text { para } & \text { la } \\
\text { recolección de } \\
\text { datos }\end{array}$ & Resultados \\
\hline $\begin{array}{l}\text { Quality of life in } \\
\text { mild dementia: } \\
\text { patterns of change } \\
\text { in self and } \\
\text { caregiver ratings } \\
\text { over time }\end{array}$ & $\begin{array}{l}\text { Marcia Dourado, } \\
\text { María de Sousa, } \\
\text { Raquel Santos, } \\
\text { José Simões } \\
\text { Neto, Marcela } \\
\text { Nogueira, } \\
\text { Tatiana Belfort, } \\
\text { Bianca Torres, } \\
\text { Rachel Días, } \\
\text { Jerson Laks. } \\
\text { Brasil, São } \\
\text { Paulo, 2016 }\end{array}$ & Estudio longitudinal. & $\begin{array}{l}\text { Escalar de } \\
\text { Calidad de Vida } \\
\text { en la } \\
\text { Enfermedad de } \\
\text { Alzheimer QoL- } \\
\text { AD. } \\
\text { Escalar de } \\
\text { Evaluación del } \\
\text { Impacto } \\
\text { Psicosocial del } \\
\text { Diagnóstico de } \\
\text { la Demencia } \\
\text { (ASPIDD). } \\
\text { Mini-Mental } \\
\text { State } \\
\text { Examination } \\
\text { (MMSE). } \\
\text { Escala de } \\
\text { Cornell para la } \\
\text { depresión en la }\end{array}$ & $\begin{array}{l}\text { Al inicio del estudio, las } \\
\text { autoevaluaciones de calidad de } \\
\text { vida de las personas con } \\
\text { demencia y sus cuidadores se } \\
\text { asociaron con las } \\
\text { clasificaciones de cuidador. } \\
\text { Las clasificaciones de los } \\
\text { cuidadores se asociaron con el } \\
\text { estado de ánimo y la calidad de } \\
\text { vida autocalificada. Después } \\
\text { de } 1 \text { año, las clasificaciones de } \\
\text { cuidadores de personas con } \\
\text { enfermedad de Alzheimer } \\
\text { cambiaron significativamente, } \\
\text { mientras que las } \\
\text { autoevaluaciones de los } \\
\text { pacientes no lo hicieron La } \\
\text { concientización sobre la la } \\
\text { enfermedad cambió } \\
\text { significativamente (p } 0.001) \\
\text { al año, disminuyó en } 25.4 \% \text { y } \\
\text { mejoró en 12.3\% de los } \\
\text { participantes. }\end{array}$ \\
\hline
\end{tabular}




\begin{tabular}{|c|c|c|c|c|}
\hline & & & $\begin{array}{l}\text { demencia } \\
\text { (CSDD). } \\
\text { Cuestionario de } \\
\text { actividades } \\
\text { funcionales de } \\
\text { Pfeffer (PFAQ). } \\
\text { El Inventario } \\
\text { Neuropsiquiátric } \\
\text { o (NPI). } \\
\text { Entrevista Zarit } \\
\text { Burden (ZBI) }\end{array}$ & $\begin{array}{l}\text { autoevaluaciones de PwD QoL } \\
\text { se asociaron con las } \\
\text { calificaciones de r los } \\
\text { cuidadores }(p=0,001) \text {. Las } \\
\text { clasificaciones de los } \\
\text { cuidadores de QoL PwD } \\
\text { después de } 1 \text { año se asociaron } \\
\text { con el estado de ánimo PwD ( } p \\
=0,029 \text { ), autoinformada ( }= \\
0,001) \text { y conocimiento de la } \\
\text { enfermedad }(p=0,033) \text {. }\end{array}$ \\
\hline $\begin{array}{lr}\text { Calidad de } & \text { vida } \\
\text { relacionada con la } \\
\text { salud } & \text { en } \\
\text { cuidadores } & \text { de } \\
\text { pacientes } & \text { con } \\
\text { enfermedad } & \text { de } \\
\text { Alzheimer. } & \end{array}$ & $\begin{array}{l}\text { Garzón } \\
\text { Maldonado A, } \\
\text { Gutiérrez- } \\
\text { Bedmar B, } \\
\text { García-Casares } \\
\text { C, Pérez } \\
\text { Errázquin } r \text { F, } \\
\text { Gallardo F y } \\
\text { Martínez Valle } \\
\text { G. } \\
\text { Málaga España, } \\
2017\end{array}$ & $\begin{array}{l}\text { Estudio longitudinal } \\
\text { pareado. }\end{array}$ & $\begin{array}{l}\text { el Cuestionario } \\
\text { de Salud SF-36; } \\
\text { La escala SF-36 } \\
\text { es un } \\
\text { cuestionario } \\
\text { genérico que } \\
\text { consta de } \\
36 \text { ítems que se } \\
\text { agrupan en } 8 \\
\text { dimensiones que } \\
\text { proporcionan } \\
\text { información } \\
\text { relativa a la } \\
\text { Función física } \\
\text { (FF), Rol físico } \\
\text { (RF), Dolor } \\
\text { corporal (DC), } \\
\text { Salud general } \\
\text { (SG), Vitalidad } \\
\text { (VT), Función } \\
\text { social (FS), Rol } \\
\text { emocional (RE), } \\
\text { Salud mental } \\
\text { (SM). }\end{array}$ & $\begin{array}{l}\text { Las } 8 \text { dimensiones de la escala } \\
\text { SF-36 en los cuidadores } \\
\text { principales empeoraron de } \\
\text { forma significativa a los } 12 \\
\text { meses, salvo las dimensiones } \\
\text { «Función física» y «Función } \\
\text { social», que lo hicieron de } \\
\text { forma no significativa. Las } \\
\text { puntuaciones en la visita basal } \\
\text { fueron menores que las } \\
\text { correspondientes a la } \\
\text { población general. La } \\
\text { dimensión que presentó peor } \\
\text { puntuación fue «Vitalidad. El } \\
\text { tiempo medio de cuidado } \\
\text { informal, en meses, a criterio } \\
\text { de los CP fue de casi arno y } \\
\text { medio (17,11 meses). Entre los } \\
\text { CP la mayoría fueron } \\
\text { hijos/hijas (61,9\%) } \\
\text { esposos/esposas (27,8\%). } \\
\text { Entre los no coetáneos había } 4 \\
\text { CP nuera-yerno, } 3 \text { CP sobrino- } \\
\text { sobrina y una nieta; entre los } \\
\text { coetáneos, una hermana y una } \\
\text { amiga. }\end{array}$ \\
\hline $\begin{array}{l}\text { Impact of caring } \\
\text { for persons with } \\
\text { Alzheimer's } \\
\text { disease or } \\
\text { dementia on } \\
\text { caregivers' health } \\
\text { outcomes: } \\
\text { findings from a } \\
\text { community based } \\
\text { survey in Japan. }\end{array}$ & $\begin{array}{l}\text { Amir Goren, } \\
\text { William , } \\
\text { Montgomery , } \\
\text { Kristin Kahle } \\
\text { Wrobleski, } \\
\text { Tomomi } \\
\text { Nakamura y } \\
\text { Kaname Ueda } \\
\text { Japón, 2016 }\end{array}$ & Estudio Descriptivo & $\begin{array}{l}\text { Cuestionario de } \\
\text { Salud del } \\
\text { Paciente (PHQ- } \\
\text { 9) que sugiere } \\
\text { presencia de } \\
\text { ausencia dastorno } \\
\text { trasta } \\
\text { depresivo mayor } \\
\text { (TDM) } \\
\text { Productividad } \\
\text { laboral } \\
\text { deterioro de la } \\
\text { actividad } \\
\text { (WPAl), calidad } \\
\text { de vida basada } \\
\text { en SF 36v2 y } \\
\text { utilización de los } \\
\text { recursos de } \\
\text { atención médica. } \\
\text { Características } \\
\text { sociodemográfic } \\
\text { as, } \\
\text { características y } \\
\text { comportamiento } \\
\text { s de salud y }\end{array}$ & $\begin{array}{l}\text { Entre los } 55060 \text { encuestados, } \\
\text { en comparación con los no } \\
\text { cuidadores ( } n=53758) \text {, los } \\
\text { cuidadores ( } n=1302) \text { eran } \\
\text { mayores }(52,6 \text { vs. } 47.5 \text { años), } \\
\text { más frecuentemente mujeres } \\
(53 \% \text { vs. 49\%), casadas / } \\
\text { parejas, bebedores frecuentes } \\
\text { de alcohol, fumadores actuales } \\
\text { y no empleados, y promediaron } \\
\text { puntajes de CCI más altos } \\
\text { (0.37 vs. 0.14), todos p <0.05. } \\
\text { Puntajes de propensión sexo } \\
\text { incorporado, edad, índice de } \\
\text { masa corporal (IMC), ejercicio, } \\
\text { alcohol, tabaquismo, estado } \\
\text { civil, ICC, estado de } \\
\text { asegurado, educación, empleo, } \\
\text { ingresos y niños en el hogar. } \\
\text { Un algoritmo de concordancia } \\
\text { codicioso produjo } 1297 \\
\text { coincidencias exactas, } \\
\text { excluyendo } 5 \text { cuidadores no } \\
\text { emparejados. Los puntajes de } \\
\text { servicios de salud fueron }\end{array}$ \\
\hline
\end{tabular}




\begin{tabular}{|c|c|c|c|c|}
\hline & & & $\begin{array}{l}\text { puntajes del } \\
\text { índice de } \\
\text { comorbilidad de } \\
\text { Charlson (CCI). }\end{array}$ & $\begin{array}{l}\text { significativamente más bajos } \\
\text { entre los cuidadores (0.724) vs. } \\
\text { No cuidadores }(0.764) \text {, al igual } \\
\text { que SF-36v2 Puntajes de } \\
\text { Resumen de Componente } \\
\text { Físico y Mental. Cuidadores vs. } \\
\text { No cuidadores tuvo un } \\
\text { ausentismo significativamente } \\
\text { mayor, deterioro relacionado } \\
\text { con el presentismo, deterioro } \\
\text { general del trabajo (25.8\% vs. } \\
20.4 \% \text { respectivamente), y } \\
\text { deterioro de la actividad }(25.4 \% \\
\text { vs. } 21.8 \%) \text { más sala de } \\
\text { emergencias y tradicional } \\
\text { visitas del proveedor ( } 7.70 \text { vs. } \\
5.35) \text { en los últimos seis } \\
\text { meses, y MDD más frecuente } \\
\text { (14\% vs. 9\%), depresión, } \\
\text { insomnio, ansiedad y dolor. }\end{array}$ \\
\hline $\begin{array}{l}\text { Benefits of } \\
\text { psychodynamic } \\
\text { group therapy on } \\
\text { depression, } \\
\text { burden and quality } \\
\text { of life of family } \\
\text { caregivers to } \\
\text { Alzheimer disease } \\
\text { patients }\end{array}$ & $\begin{array}{l}\text { Dorli Kamkhagi, } \\
\text { Ana Carolina } \\
\text { Oliveira Costa, } \\
\text { Sandra } \\
\text { Kusminsky, } \\
\text { Deborah Supino, } \\
\text { Breno Satler } \\
\text { Diniz, Wagner } \\
\text { Farid Gattaz y } \\
\text { Orestes Vicente } \\
\text { Forlenza } \\
\text { São Paulo, } \\
\text { Brasil, 2015 }\end{array}$ & $\begin{array}{l}\text { Ensayo } r \text { de } \\
\text { intervención } \\
\text { controlado simple } \\
\text { ciego. }\end{array}$ & $\begin{array}{l}\text { Las medidas de } \\
\text { resultado fueron: } \\
\text { modificación de } \\
\text { puntajes en Zarit } \\
\text { Burden Scale, } \\
\text { Beck Depression } \\
\text { Inventory y } \\
\text { WHO-QoL } \\
\text { Scale. }\end{array}$ & $\begin{array}{l}\text { Los participantes en el grupo } \\
\text { PGT (Psicoterapia grupal } \\
\text { psicodinámica) mostraron una } \\
\text { reducción significativa en la } \\
\text { carga }(p=0,01) \text { y los puntajes } \\
\text { de depresión }(p=0,005) \text { y una } \\
\text { mejor calidad de vida ( } p= \\
0,002) \text { mientras que los del } \\
\text { grupo BAT (Terapia de } \\
\text { conciencia corporal) mostraron } \\
\text { mejoras en la carga de la } \\
\text { atención }(p=0.001) \text { y calidad } \\
\text { de vida }(p=0.01), \text { pero no en } \\
\text { síntomas depresivos }(p=0.13) \text {. }\end{array}$ \\
\hline $\begin{array}{l}\text { Social skills and } \\
\text { well-being among } \\
\text { family caregivers } \\
\text { to patients with } \\
\text { Alzheimer's } \\
\text { disease. }\end{array}$ & $\begin{array}{l}\text { Flávia Araujo de } \\
\text { Amorim, } \\
\text { Mariana de } \\
\text { Campos Pereira } \\
\text { Giorgion, } \\
\text { Orestes Vicente } \\
\text { Forlenz } \\
\text { São Paulo, } \\
\text { Brasil, 2017 }\end{array}$ & Estudio Descriptivo & \begin{tabular}{lr} 
Social & Skills \\
Inventory & (SSI- \\
Del-Prette) & y la \\
entrevista & Zarit \\
Burden; $\quad$ la \\
calidad de vida \\
se estimó con el \\
cuestionario \\
\multicolumn{2}{l}{ WHO-QoL- bref. }
\end{tabular} & $\begin{array}{l}\text { Encontramos correlaciones } \\
\text { positivas entre las } \\
\text { puntuaciones totales de SSI y } \\
\text { los dominios psicológicos }(r= \\
0,450 ; p=0,003 \text { ) y ambientales } \\
(r=0,408 ; p=0,008) \text { de WHO- } \\
\text { QoL- bref. El factor SSI } \\
\text { 'autocontrol de la agresividad' } \\
(\mathrm{SSI}-\mathrm{F} 5) \text { se correlacionó } \\
\text { negativamente con la magnitud } \\
\text { de la carga del cuidador }(r=- \\
0,483 ; p=0,001) \text { y se asoció } \\
\text { positivamente con el dominio } \\
\text { psicológico de WHO-QoL- bref } \\
(r=0.446 ; p=0.003) \text { Los } p \\
\text { cuidadores con una mejor } \\
\text { "autoafirmación en la expresión } \\
\text { del afecto positivo" (SSI-F2) } \\
\text { también tenían mejores } \\
\text { "relaciones sociales" según } \\
\text { WHO-QoL- bref }(r=0,402 ; p= \\
0,009) \text { SSI: inventario de } \\
\text { habilidades sociales; F1: } \\
\text { afrontamiento y autoafirmación } \\
\text { con riesgo; F2: autoafirmación } \\
\text { en la expresión del afecto } \\
\text { positivo; F3: conversación y } \\
\text { confianza social; F4: auto } \\
\text { exposición a personas } \\
\text { desconocidas y situaciones }\end{array}$ \\
\hline
\end{tabular}




\begin{tabular}{|c|c|c|c|c|}
\hline & & & & $\begin{array}{l}\text { nuevas; F5: autocontrol de la } \\
\text { agresividad. }\end{array}$ \\
\hline $\begin{array}{lr}\text { Stress and quality } \\
\text { of life of the family } \\
\text { caregivers of } \\
\text { elderly } & \text { of } \\
\text { Alzheimer's } & \\
\text { disease } & \end{array}$ & $\begin{array}{l}\text { Vanovya Alves } \\
\text { Claudino } \\
\text { Cesário, Márcia } \\
\text { Carréra Campos } \\
\text { Leal, Ana Paula } \\
\text { de Oliveira } \\
\text { Marque, } \\
\text { Karolyny Alves } \\
\text { Claudino } \\
\text { Pernanbuco, } \\
\text { Brasil, 2017. }\end{array}$ & $\begin{array}{l}\text { Investigación de } \\
\text { abordaje } \\
\text { cuantitativo del tipo } \\
\text { descriptivo. }\end{array}$ & $\begin{array}{lr}\text { Se aplicó } & \text { el } \\
\text { Inventario } & \text { de } \\
\text { Síntomas } & \text { de } \\
\text { Estrés } & \text { para } \\
\text { Adultos } \quad \text { Lipp } \\
\text { (ISSL) y la } \\
\text { versión brasileña } \\
\text { de la Calidad de } \\
\text { Vida } \\
\text { Cuestionario de } \\
\text { Salud Short } \\
\text { Form (SF-36) en } \\
\text { portugués. }\end{array}$ & $\begin{array}{l}\text { Los cuidadores familiares de } \\
\text { ancianos evaluados en este } \\
\text { estudio presentaron un } \\
\text { promedio de edad de } 56,98 \\
\text { años (dp }=11,39 \text { ), tiempo } \\
\text { promedio de cuidado de } 4,64 \\
\text { años (dp }=2,98) \text {, con } 55,8 \% \\
\text { ejerciendo la función de } \\
\text { cuidador desde hace más de } 3 \\
\text { años, habiendo predominancia } \\
\text { del sexo femenino (n = } 37 \text {; } \\
86 \%) ; \text { en cuanto al grado de } \\
\text { parentesco con el anciano, } \\
\text { había } 33 \text { (76,8\%) esposas o } \\
\text { hijas; } 27 \text { participantes (62,8\%) } \\
\text { poseían vínculo laboral; y } 16 \\
\text { (37,2\%) indicaron presentar } \\
\text { alguna morbilidad. } \\
\text { Entre los dominios que } \\
\text { expresan factores relacionados } \\
\text { a la calidad de vida, se } \\
\text { destacan las reducidas medias } \\
\text { en vitalidad (48,84) y aspectos } \\
\text { sociales (56,69). Entre los } \\
\text { síntomas predominantes que } \\
\text { favorecen el marco de estrés, } \\
16 \text { (59,3\%) tenían cuidadores } \\
\text { familiares sintomatología } \\
\text { psicológica en la costa de la } \\
\text { física. }\end{array}$ \\
\hline $\begin{array}{l}\text { Assessment of } \\
\text { Health-Related } \\
\text { Quality of Life for } \\
\text { Caregivers of } \\
\text { Alzheimer's } \\
\text { Disease Patients }\end{array}$ & $\begin{array}{l}\text { Maria } \\
\text { Andreakou, } \\
\text { Angelos } \\
\text { Papadopoulos, } \\
\text { Demóstenes } \\
\text { Panagiotakos y } \\
\text { Dimitris Niakas } \\
\text { Grecia, 2016. }\end{array}$ & $\begin{array}{l}\text { Se trata de un } \\
\text { estudio } \\
\text { observacional } \\
\text { diseñado para } \\
\text { evaluar } \\
\text { características } \\
\text { descriptivas y y } \\
\text { asociaciones entre } \\
\text { parámetros } \\
\text { sociodemográficos } \\
\text { y emocionales con } \\
\text { el tiempo dedicado } \\
\text { a la atención de } \\
\text { pacientes con EA. }\end{array}$ & \begin{tabular}{lr}
\multicolumn{3}{l}{ Cuestionario } \\
estructurado y \\
cercano para \\
recuperar la \\
información lán \\
sociodemográfic \\
a y familiar de \\
los participantes. \\
Escala ra de \\
Depresión de \\
Zung (ZDRS). \\
Entre \\
diferentes los \\
cuestionarios \\
para evaluar la \\
CVRS, r la \\
Encuesta de \\
Salud de Forma \\
Corta 36 (SF- \\
36).
\end{tabular} & $\begin{array}{l}\text { Se reveló una fuerte } \\
\text { correlación negativa entre las } \\
\text { dimensiones de la CVRS y los } \\
\text { puntajes de la escala de } \\
\text { depresión. Los cuidadores de } \\
\text { pacientes con EA tienen una } \\
\text { CVRS menor en casi todas las } \\
\text { dimensiones en comparación } \\
\text { con la población general } \\
\text { urbana griega. El papel social } \\
\text { de los cuidadores, la existencia } \\
\text { de problemas emocionales y su } \\
\text { estado de salud mental } \\
\text { condujeron a este resultado. } \\
\text { Además, se indicaron } \\
\text { diferencias significativamente } \\
\text { importantes en la CVRS total y } \\
\text { la sintomatología depresiva de } \\
\text { los cuidadores en relación con } \\
\text { su género, existencia de } \\
\text { hipertensión, frecuencia de } \\
\text { atención al paciente, } \\
\text { cohabitación con el paciente, } \\
\text { agravamiento de la } \\
\text { enfermedad y estado } \\
\text { económico. y de }\end{array}$ \\
\hline
\end{tabular}

De los estudios seleccionados para esta revisión, la mayor parte se realizaron en Latinoamérica, $57 \%$ de los estudios se llevaron a cabo en Brasil y en un porcentaje similar de 14,2\% en España, Japón y Grecia. El período de tiempo que se contempló fue los últimos 5 años, encontrando que el $42,9 \%$ corresponden al año 2017 y un mismo porcentaje al año 2016, mientras que el 14,2\% corresponde al año 2015.

En relación a la muestra de los estudios seleccionados, decir que el número total de cuidadoras/es incluidos en las investigaciones revisadas fue de 1,744. En cuanto a la composición de la muestra, el $85,7 \%$ de los estudios sólo incluían cuidadoras/es de pacientes con enfermedad de Alzheimer y un 14,2\% incluía el 
binomio de calidad de vida paciente-cuidador.

Respecto a la calidad de vida como tal, sólo un estudio midió de manera directa la calidad de vida de los cuidadores/as de personas con enfermedad de Alzheimer y describe que es la calidad de vida, otro estudio comparó la calidad de vida del cuidador/a de pacientes con enfermedad de Alzheimer versus el no cuidador/a, mientras que un estudio midió la calidad de vida de los cuidadores/as como resultado secundario. Tres la relacionaron como la consecuencia de depresión, nivel de estrés, el tiempo de evolución de la enfermedad y para medir la efectividad de dos intervenciones de apoyo psicológico.

Los estudios utilizaron varios instrumentos para medir la calidad de vida y las variables independientes de los cuidadores/as de personas con enfermedad de Alzheimer como: fueron escala de Calidad de Vida en la Enfermedad de Alzheimer (QoL-AD); La entrevista Zarit Burden (ZBI) para la carga del cuidador, para medir el bienestar del cuidador/a el Cuestionario de Salud SF36 , cuyos ítems se agrupan en 8 dimensiones que proporcionan información relativa a la Función física, Rol físico, Dolor corporal, Salud general, Vitalidad, Función social, Rol emocional, Salud mental. El Cuestionario de Salud del Paciente (PHQ-9) que sugiere presencia / ausencia de trastorno depresivo mayor (TDM) Productividad laboral y deterioro de la actividad (WPAI), este instrumento permitió determinar la relación existente con la calidad de vida. También se aplicó la escala de Beck Depression Inventory, para medir el nivel de depresión de los cuidadores/as, el Inventario de Síntomas de Estrés para Adultos LIPP (ISSL) y el cuestionario estructurado para la información sociodemográfica y familiar de los participantes.

Entre los tipos de estudio utilizados por las investigaciones revisadas correspondieron en un mayor porcentaje a los de tipo descriptivo 42,8\%; seguido por los de tipo longitudinal en un $28,5 \%$; un estudio descriptivo - observacional que corresponde al $14,2 \%$ y un ensayo de intervenciones controlado de simple ciego en un $14,2 \%$.

De acuerdo con los estudios revisados se pudo evidenciar que la mayor parte de cuidadores/as son parte de la familia 0 allegados cercanos a la persona con enfermedad de Alzheimer, los cuidados que ellos proveen no están regulados, ni sometidos a horarios, ni remuneración económica y generalmente quienes lo proveen han tenido una educación formal, lo cual facilita el desempeño del rol y que la mayor parte de personas con enfermedad de Alzheimer viven en su hogar.

El cuidado de la persona con enfermedad de Alzheimer por lo general recae en mayor número en el conyugue y al mismo tiempo está relacionada con el género del enfermo, la mayor parte de cuidadores son mujeres, así como también se menciona que con frecuencia los cuidadores tienen necesidad de asistencia médica, principalmente con trastornos relacionados a la parte psicológica y emocional debido a un alto índice de depresión, estrés, incertidumbre y una larga exposición al cuidado. El estado de salud y bienestar del enfermo determina de manera directa la calidad de vida que tiene su cuidador/a.

\section{DISCUSIÓN}

El cuidado de la persona con enfermedad de Alzheimer es una tarea compleja. El cuidador es aquella persona que desde el inicio de la enfermedad asume la asistencia y cuidados diarios del enfermo suministrando de forma directa la ayuda que este necesita, a medida que la enfermedad va deteriorando sus capacidades funcionales y autonomía.

El cuidado en la vida del cuidador tiene una repercusión negativa en su salud, debido al aparecimiento de afecciones físicas, psicológicas, socioeconómicas principalmente. A través de esta revisión integrativa se ha podido evidenciar cómo es la calidad de vida de los cuidadores de personas con enfermedad de Alzheimer.

\section{Perfil de los cuidadores de personas con enfermedad de Alzheimer}

Goren et al. 2017 (18); en su estudio resalta la importancia de caracterizar a los cuidadores de personas con enfermedad de Alzheimer, para conocer sus necesidades, identificar sus problemas y para evaluar la efectividad de las intervenciones que han sido ejecutadas en esta población. Además Los autores describen las características de los cuidadores de personas con enfermedad de Alzheimer y la relación entre el acto de cuidar y la salud de los mismos $(14,19)$. Con relación al género se encontró que el mayor porcentaje de cuidadores/as son mujeres 18. Otros estudios corroboraron con este hallazgo $(19,20,21)$. La asignación del cuidado en el núcleo familiar también está determinado en función del género de la persona enferma y su rol familiar, si es hombre el cuidado es asumido por el conyugue (22), si es mujer el cuidado es asumido por sus hijas o personas allegadas a la familia (14). Además sostienen que las mujeres son más vulnerables a sufrir restricciones sociales a causa del cuidado y experimentan mayor grado de sobrecarga (21).

Según los estudios las edades comprendidas de los cuidadores/as en su gran mayoría se situaron sobre los 57 años (21), debido a que el cuidado recae en el conyugue en mayor porcentaje (14). Es por ello que se desprende que un número importante de ancianos que cuidan a otros iguales (18). Otros estudios corroboran con estas afirmaciones y señalan que los cuidadores con edad avanzada probablemente experimentan mayor nivel de sobrecarga que los cuidadores jóvenes $(14,23)$. De acuerdo con los datos correspondientes a la situación socioeconómica de los cuidadores Goren et al. 2017 (18), hace referencia sobre la importancia y el impacto de la situación financiera que enmarca el cuidado del paciente con enfermedad de Alzheimer, principalmente por los medicamentos, controles y terapias sanitarias, movilidad, entre otros.

Los resultados de este estudio identificaron el predominio de cuidadores con educación formal, lo cual facilita la comprensión sobre la enfermedad y contribuye con la disminución de los trastornos depresivos y estrés en los cuidadores $(14,20,21)$. Sin embargo, Daurado et al. 2016 (23) reconoce en su estudio que los cuidadores que informaron bajos niveles de calidad de vida tienen un conocimiento deficiente de la enfermedad, también mencionan que la calidad de vida del cuidador está directamente relacionada con la estabilidad del estado de ánimo, ya que puede haber llevado a evaluaciones de calidad de vida más positivas en el cuidador. Además, el estado de ánimo de las personas con enfermedad de Alzheimer tiene un impacto en las calificaciones de los cuidadores sobre la calidad de vida.

Tener mayor experiencia en el cuidado mejora la percepción de bienestar en la persona enferma, debido a que existe un dominio del cuidado con el transcurso del tiempo, pero al mismo tiempo existen más posibilidades de desarrollar estrés o problemas emocionales que influyen en la calidad de vida del cuidador (21).

\section{Calidad de vida del cuidador de personas con enfermedad de Alzheimer}

La calidad de vida es la forma como el individuo percibe su vida, el lugar que ocupa en el contexto en que vive, la relación con sus objetivos, expectativas, normas, criterios y preocupaciones, todo ello permeado por las actividades diarias, la salud física, el estado psicológico, el grado de independencia, las relaciones sociales, los factores ambientales y sus creencias personales, 
los cuales están relacionadas con la calidad de vida (13).

La calidad de vida del cuidador se ve afectada porque tiene que luchar con el paciente a lo largo de su vida (24), uno de los factores determinantes que inciden en la calidad de vida del cuidador/a es el éxito de la adaptación y afrontamiento al papel del cuidador $(14,21,22)$.

Para Kamkhagi y Olivera el al. 2017 (24). Muestran que la progresión de la enfermedad, el deterioro cognitivo y funcional causa en la persona enferma la pérdida de autonomía y una mayor dependencia para realizar actividades básicas de la vida diaria, que requieren de cuidados y genera un impacto en los cuidadores que tendrán que dedicar más tiempo para el cuidado del paciente, bajo altos niveles de estrés, angustia psicológica relacionada con el nuevo rol, fatiga crónica, por las horas prolongadas de asistencia, sin períodos de descanso y sueño adecuado.

Entre las principales alteraciones en la salud de los cuidadores/as de personas con enfermedad de Alzheimer están las alteraciones físicas, psicológicas y emocionales, sociales y financieras, que desencadenan mayor riesgo de comorbilidad, deterioro de la productividad y mayores tasas de uso de recursos sanitarios $(14,19,22,24)$.

Según Goren el al. 2017 (18), establecen que entre los trastornos psicológicos frecuentes están la ansiedad, angustia emocional y depresión, lo que puede afectar aún más la capacidad del cuidador. La preservación de la salud física y mental de los cuidadores de pacientes con enfermedad de Alzheimer debe entenderse como un activo para alcanzar el cumplimiento eficaz y ético de este rol fundamental $(18,21)$.

El estado emocional de los cuidadores/as de personas con enfermedad de Alzheimer (24), tiene un impacto en las calificaciones de los cuidadores sobre la calidad de vida., porque le permiten dar respuestas a las múltiples demandas que surgen en el largo y deteriorado curso de la enfermedad, en la finalización de un rol que no siempre valorado como debería ser por otros miembros de la familia $(19,22,23)$.

\section{Apoyo social}

La feminización del cuidado de paciente con Enfermedad de Alzheimer, sumado al incremento de más personas mayores cuidando de otras personas de igual o mayor edad ocasiona que la carga del cuidado sea más difícil y por lo tanto los cuidadores/as necesitan recibir apoyo y atención $(14,19,24)$.
Según los estudios en la mayoría de veces el cuidador ha tenido tener que enfrentarse al diagnóstico de la enfermedad y al cuidado de la persona enferma sin orientación, ni ayuda pertinente, sin apoyo social, lo que interfieren en su calidad de vida $(14,21,22)$. Por lo tanto el equipo de salud necesita implementar acciones que contemplen las necesidades de los cuidadores, tales como directrices que orienten la práctica del cuidado de este tipo especial de enfermos, mejorar la asistencia a la salud, apoyo físico y emocional de los cuidadores/as (21). Además, es necesario producir y hacer efectivas políticas públicas que amparen y faciliten a los cuidadores, cuidar de sus ancianos con dignidad, amor, con un nivel de sobrecarga bajo, que promueva una excelente calidad de vida.

\section{CONCLUSIÓN}

Por medio de este estudio se pudo analizar cuál es la calidad de vida de los cuidadores/as de personas con enfermedad de Alzheimer, visualizando que la responsabilidad del cuidado en mayor parte recae en la familia y que dentro de los cuidadores el mayor porcentaje son mujeres. Además, se pudo evidenciar que la calidad de vida de los cuidadores está intrínsecamente relacionada con factores como bienestar del enfermo, estrés, depresión, tiempo de exposición al cuidado, carga física y emocional; todas estas condiciones pueden influir directamente en el cuidado prestado.

Es importante que los estudios permitan una comprensión integral de la calidad de vida de los cuidadores de personas con enfermedad de Alzheimer, bajo una mirada amplia sobre que saque a flote sus necesidades, comorbilidades y problemas relacionados con el cuidado, que favorezcan el planteamiento de estrategias, acciones de promoción y prevención de salud dirigida a los cuidadores. Con ello se percibe una necesidad creciente de que los profesionales de salud y en especial enfermería realice intervenciones enfocadas al acompañamiento, educación y apoyo de las necesidades en esta población tan vulnerable.

\section{CONFLICTO DE INTERESES}

Las autoras declaran no tener conflicto de intereses con personas o instituciones de tipo: académicas, comerciales, políticas o financieras.

\section{REFERENCIAS}

1. Ilha S, Backes DS, Santos SSC, Gautério-Abreu DP, Silva BT da, Pelzer MT, et al. Alzheimer's disease in elderly/family: Difficulties experienced and care strategies. Esc Anna Nery. marzo de 2016;20(1):138-146.

2. Kucmanski LS, Zenevicz L, Geremia DS, Madureira VSF, Silva TG da, Souza SS de, et al. Alzheimer's desease: challenges faced by family caregivers. Rev Bras Geriatr E Gerontol. diciembre de 2016;19(6):1022-9.

3. Marins AM da F, Hansel CG, da Silva J, Marins AM da F, Hansel CG, da Silva J. Behavioral changes of elderly with Alzheimer's Disease and the burden of care for the caregiver. Esc Anna Nery. junio de 2016;20(2):352-356.

4. OMS. Estrategia y plan de acción sobre demencias en las personas mayores [Internet]. 2015. Disponible en: https://www.paho.org/hq/dmdocuments/2015/CD54-8-s.pdf

5. Puchi C, Jara P, Puchi C, Jara P. Enfermería y el cuidado domiciliario de los mayores en la era de la globalización. Enferm Univ. 2015;12(4):219-225.

6. Malvárez S. El reto de cuidar en un mundo globalizado. Texto Amp Contexto - Enferm. septiembre de 2007;16(3):520-30.

7. OMS. Demencia [Internet]. 2016 [citado 15 de mayo de 2018]. Report No.: 139. Disponible en: http://apps.who.int/gb/ebwha/pdf_files/EB139/B139_3-sp.pdf

8. Amador-Marín B, Guerra-Martín MD. Eficacia de las intervenciones no farmacológicas en la calidad de vida de las personas cuidadoras de pacientes con enfermedad de Alzheimer. Gac Sanit. abril de 2017;31:154-160.

9. Pérez Rodríguez M, Álvarez Gómez T, Badaló M, Javier E, Valdivia Cañizares S, Borroto Carpio I, et al. El síndrome del cuidador en cuidadores principales de ancianos con demencia Alzhéimer. Gac Médica Espirituana. abril de 2017;19(1):38-50.

10. Weltgesundheitsorganisation, Alzheimer's Disease International, editores. Dementia: a public health priority. Geneva; 2012. $102 \mathrm{p}$. 
11. Leiva Díaz V, Hernández Rojas ME, Aguirre Mora E. Experiencias de familias que conviven con una persona con diagnóstico de Alzheimer. Enferm Actual Costa Rica [Internet]. 31 de diciembre de 2015;(30). Disponible en: http://revistas.ucr.ac.cr/index.php/enfermeria/article/view/22550

12. Carreño-Moreno SP, Chaparro-Díaz L. Calidad de vida de los cuidadores de personas con enfermedad crónica. Aquichan. 1 de diciembre de 2016;16(4):447-461.

13. Inouye K, Pedrazzani ES, Pavarini SCI, Toyoda CY. Quality of life of elderly with alzheimer's disease: a comparative study between the patient's and the caregiver's report. Rev Lat Am Enfermagem. febrero de 2010;18(1):26-32.

14. Garzón-Maldonado FJ, Gutiérrez-Bedmar M, García-Casares N, Pérez-Errázquin F, Gallardo-Tur A, Martínez-Valle Torres MD. Calidad de vida relacionada con la salud en cuidadores de pacientes con enfermedad de Alzheimer. Neurología. octubre de 2017;32(8):508-515.

15. Pérez Rodríguez M, Álvarez Gómez T, Badaló M, Javier E, Valdivia Cañizares S, Borroto Carpio I, et al. El síndrome del cuidador en cuidadores principales de ancianos con demencia Alzhéimer. Gac Médica Espirituana. abril de 2017;19(1):38-50.

16. Roldán Ahedo N, Corral Liria I. Intervenciones de enfermería para la atención del cuidador informal del paciente con Alzheimer. Revisión bibliográfica. Rev Rol Enferm. 2017;40(10):690-697.

17. Orellana Yañez A, Paravic Klijn T. Enfermería basada en evidencia: barreras y estrategias para su implementación. Cienc Enferm. junio de 2007;13(1):17-24.

18. Goren A, Montgomery W, Kahle-Wrobleski K, Nakamura T, Ueda K. Impact of caring for persons with Alzheimer's disease or dementia on caregivers' health outcomes: findings from a community based survey in Japan. BMC Geriatr [Internet]. diciembre de 2016;16(1). Disponible en: http://bmcgeriatr.biomedcentral.com/articles/10.1186/s12877-016-0298-y

19. Andreakou MI, Papadopoulos AA, Panagiotakos DB, Niakas D. Assessment of Health-Related Quality of Life for Caregivers of Alzheimer's Disease Patients. Int J Alzheimers Dis [Internet]. 2016;2016. Disponible en: https://www.ncbi.nlm.nih.gov/pmc/articles/PMC5204092/

20. Amorim FA de, Giorgion M de CP, Forlenza OV, Amorim FA de, Giorgion M de CP, Forlenza OV. Social skills and well-being among family caregivers to patients with Alzheimer's disease. Arch Clin Psychiatry São Paulo. diciembre de 2017;44(6):15961.

21. Cesário VAC, Leal MCC, Marques AP de O, Claudino KA, Cesário VAC, Leal MCC, et al. Stress and quality of life of the family caregivers of elderly with Alzheimer's disease. Saúde Em Debate. marzo de 2017;41(112):171-82.

22. Kamkhagi D, Costa ACO, Kusminsky S, Supino D, Diniz BS, Gattaz WF, et al. Benefits of psychodynamic group therapy on depression, burden and quality of life of family caregivers to Alzheimer disease patients. Arch Clin Psychiatry São Paulo. diciembre de 2015;42(6):157-160.

23. Dourado MC, Sousa MF de, Santos RL, Simões Neto JP, Nogueira ML, Belfort TT, et al. Quality of life in mild dementia: patterns of change in self and caregiver ratings over time. Rev Bras Psiquiatr. 8 de enero de 2016;38(4):294-300.

24. Kamkhagi D, Costa ACO, Kusminsky S, Supino D, Diniz BS, Gattaz WF, et al. Benefits of psychodynamic group therapy on depression, burden and quality of life of family caregivers to Alzheimer's disease patients. Arch Clin Psychiatry São Paulo. diciembre de 2015;42(6):157-160. 